\title{
Time-Resolved Diffuse Optical Spectroscopy: A Differential Absorption Approach
}

\author{
PAOLA TARONI,* ANDREA BASSI, LORENZO SPINELLI, RINALDO CUBEDDU, \\ and ANTONIO PIFFERI
}

Dipartimento di Fisica, Politecnico di Milano, piazza Leonardo da Vinci 32, 20133 Milan, Italy (P.T., A.B., R.C., A.P.); Istituto di Fotonica e
Nanotecnologie, Consiglio Nazionale delle Ricerche (P.T., L.S.); National Laboratory for Ultrafast and Ultraintense Optical Science, Consiglio
Nazionale delle Ricerche (A.B., R.C., A.P.); and Research Unit Politecnico di Milano, Istituto Italiano di Tecnologia (R.C., A.P.)

\begin{abstract}
A method is presented for the estimate of spectral changes in the absorption properties of turbid media from time-resolved diffuse optical spectroscopy. The method relies on the hypothesis of constant scattering over the wavelength range of interest, but no limitations come from the sample size and shape as the method is derived directly from the BeerLambert law. The effects of a moderate spectral dependence of the scattering properties and of the non-ideal instrument response function were investigated theoretically, and the results were confirmed experimentally, showing that the method can be profitably applied in cases of practical interest.
\end{abstract}

Index Headings: Absorption spectroscopy; Beer-Lambert law; Photon migration; Turbid media.

\section{INTRODUCTION}

The optical properties of a medium depend on its composition and microscopic structure. A wealth of information on the medium can be gained from their assessment. Thus, optical techniques, which are also inherently noninvasive, are presently applied or investigated for their potential use as diagnostic means in a variety of fields, including-but not limited to-biology and medicine.

Most often, the optical parameter of direct interest is the absorption, because its estimate at multiple wavelengths allows one to derive the composition of the medium under investigation. However, in many real situations, the absorption properties cannot be easily assessed by conventional means due to the strong scattering. When highly diffusive media are involved, operation in the time domain allows one to disentangle the absorption from the scattering contribution to light attenuation, provided that light propagation can be correctly modeled for the system under study. In most cases, in order to deal with an analytical solution of the problem, experimental data are interpreted using simple models that hold accurately only for infinite or semi-infinite media and typically rely on the diffusion approximation to the radiative transfer equation (see, for example, Ref. 1).

An analytical solution of the radiative transfer equation itself has also been derived, but still for an infinite ${ }^{2}$ or semi-infinite medium. ${ }^{3}$ On the other hand, lately the diffusion approximation has also been extended to provide straightforward close-form solutions for specific sample geometries. ${ }^{4-7}$ These models can be effectively applied in several situations, but various conditions of practical interest exist that are far from fulfilling their hypotheses. This typically occurs for samples of irregular shape and/or very small size.

Received 26 March 2010; accepted 5 August 2010.

* Author to whom correspondence should be sent. E-mail: paola.taroni@ fisi.polimi.it.
As mentioned above, optical techniques (ranging from Raman to vibrational spectroscopy) are often investigated for their potential diagnostic use or are even already applied effectively. As an example, this occurs when agricultural produce (either fruits or vegetables) is probed for nondestructive quality evaluation, including nondestructive firmness testing and the assessment of sugars related to sweetness and ripening, or of carotenoid levels to provide an indication of oxidative deterioration. ${ }^{8,9}$ Time-domain photon migration techniques have recently shown good promise in the field, ${ }^{10,11}$ but the irregular shape of the samples can limit their accuracy in the estimate of the optical properties and consequently the potential to develop effective quantitative diagnostics.

Furthermore, even samples characterized by cylindrical or parallelepiped geometry cannot be accurately modeled if their size is too small. ${ }^{6}$ This is typically the case for pharmaceutical tablets that need to be checked for content uniformity. Conventional quality control methods such as high-performance liquid chromatography (HPLC) and mass spectroscopy (MS) are time consuming, expensive, and require sample preparation. Even more important, they are destructive, so that only small samples may be tested from given production batches. Furthermore, they do not provide any information about the spatial distribution of components within a sample. Chemical imaging, which combines conventional imaging and vibrational spectroscopy, is thus emerging for process monitoring and control at all stages, from raw material to packaged product characterization. ${ }^{12,13}$ Near-infrared andmore generally - optical techniques require no sample preparation, are fast and cheap, and provide capacity for remote measurements through fiber-optic probes. Thus, they could prove very effective if quantitative results were obtained in real measurement conditions.

Several years ago an experimental approach relying on the Beer-Lambert law was introduced, specifically for use in oximetry. Its aim was the estimate of blood content and oxygenation level in living tissue from time-resolved reflection measurements performed at two wavelengths. ${ }^{14}$ The present work is essentially founded on the same theoretical base, the Beer-Lambert law, to derive the spectral dependence of the absorption coefficient from time-resolved reflection measurements performed over a certain wavelength range. Because the Beer-Lambert law comes directly from the radiative transport equation, the proposed method is not affected by the hypotheses that typically limit the application of the diffusion approximation for an infinite/semi-infinite medium, such as high albedo or sample size large enough to make boundary effects negligible. However, a strong assumption is made that the scattering properties should not change with wavelength. Thus, the performances of the proposed method were tested 
against the diffusion equation and experimental measurements were carried out to investigate the dependence on sample optical and physical properties (i.e., scattering and size, respectively) and experimental parameters (i.e., instrument response function).

\section{THEORY AND SIMULATIONS}

Estimate of the Absorption Coefficient $\boldsymbol{\mu}_{\mathrm{a}}(\boldsymbol{\lambda})$. In reflection geometry, the impulse response of a homogeneous medium, i.e., the time distribution of the reemitted light upon injection of a $\delta$-like light pulse, can be modeled as:

$$
R(\lambda, t)=A(\lambda) \varphi\left(\mu_{\mathrm{s}}^{\prime}, t, \rho\right) \exp \left(-\mu_{\mathrm{a}} v t\right)
$$

where $\mu_{\mathrm{a}}$ and $\mu_{\mathrm{s}}^{\prime}$ are the absorption and reduced scattering coefficients of the medium, $v$ is the photon velocity in the medium, and $\rho$ is the separation between light injection and collection points on the medium surface. As explicitly shown, $\varphi$ is independent of the absorption coefficient, while it depends on the scattering properties and measurement conditions through the parameter $\rho$. Moreover, it is affected by the boundary conditions and medium shape. The parameter $A$ accounts for the spectral dependence of the detection efficiency. Obviously, also the optical parameters $\mu_{\mathrm{a}}$ and $\mu_{\mathrm{s}}^{\prime}$ may change with wavelength.

Our aim is to estimate the wavelength dependence of the absorption properties over a selected spectral range from timeresolved reflection (or transmission) measurements performed as a function of wavelength.

Let us make the hypothesis that the scattering properties can be regarded as wavelength independent in the spectral range of interest. From Eq. 1, we can calculate the logarithmic ratio $S\left(\lambda_{0}, \lambda, t\right)$ of the reemitted light at two wavelengths $\lambda_{0}$ and $\lambda$ :

$$
\begin{aligned}
S\left(\lambda_{0}, \lambda, t\right) & =\ln \left[\frac{R(\lambda, t)}{R\left(\lambda_{0}, t\right)}\right] \\
& =\ln \left[\frac{A(\lambda) \varphi\left(\mu_{\mathrm{s}}^{\prime}, t, \rho\right)}{A\left(\lambda_{0}\right) \varphi\left(\mu_{\mathrm{s}}^{\prime}, t, \rho\right)}\right]-\Delta \mu_{\mathrm{a}}\left(\lambda_{0}, \lambda\right) v t \\
& =\ln \left[\frac{A(\lambda)}{A\left(\lambda_{0}\right)}\right]-\Delta \mu_{\mathrm{a}}\left(\lambda_{0}, \lambda\right) v t
\end{aligned}
$$

where $\Delta \mu_{\mathrm{a}}\left(\lambda_{0}, \lambda\right)=\mu_{\mathrm{a}}(\lambda)-\mu_{\mathrm{a}}\left(\lambda_{0}\right)$ is the absorption change between $\lambda_{0}$ and $\lambda$.

If the absorption coefficient is known a priori at a single wavelength $\lambda_{0}$, then the absolute values of the absorption coefficient as a function of wavelength, namely the absorption spectrum, can be derived over the entire spectral range.

$S\left(\lambda_{0}, \lambda, t\right)$ depends linearly on time $t$, and its slope, estimated from the best fit to a straight line, provides an easy estimate of $\Delta \mu_{\mathrm{a}}\left(\lambda_{0}, \lambda\right)$ that is totally independent of the logarithmic amplitude ratio. If $\mu_{\mathrm{a} 0}=\mu_{\mathrm{a}}\left(\lambda_{0}\right)$ is known, the absorption coefficient $\mu_{\mathrm{a}}(\lambda)$ [otherwise the absorption change $\Delta \mu_{\mathrm{a}}\left(\lambda_{0}, \lambda\right)$ ] is obtained at $N$ different wavelengths by applying Eq. 2 for each of the distinct wavelengths, thus dealing with $N$ independent linear equations.

Alternatively, if $\mu_{\mathrm{a} 0}$ is not known a priori, but the absorption properties (namely the extinction coefficient as a function of wavelength) of the constituents are known, the absolute absorption spectrum of the medium can be derived by means of a spectrally constrained fitting procedure relying on the Beer law where the constituent concentrations are free parameters. ${ }^{15}$

Errors in the Estimate of $\mu_{\mathrm{a}}(\lambda)$ : Effect of a Change in Scattering with Wavelength. Other than the assumption of homogeneous medium, the only strong and potentially limiting hypothesis we made to derive Eq. 2 is that the scattering properties are wavelength independent in the spectral range of interest $\left[\mu_{\mathrm{s}}^{\prime}(\lambda)=\mu_{\mathrm{s}}^{\prime}\left(\lambda_{0}\right)=\mu_{\mathrm{s} 0}^{\prime}\right.$ ]. In practice, this occurs with good approximation when close wavelengths are considered and for media characterized by large scattering centers that lead to a rather flat scattering spectrum. In other cases, spectral changes in scattering affect the estimate of the absorption properties.

To derive a straightforward estimate of the error introduced by scattering changes, we consider the simplifying hypothesis of an infinite homogeneous medium that leads to

$$
\varphi(\lambda, t)=A(\lambda) v\left(\frac{4 \pi v t}{3 \mu_{\mathrm{s}}^{\prime}}\right)^{-\frac{3}{2}} \exp \left(-\frac{3 \rho^{2} \mu_{\mathrm{s}}^{\prime}}{4 v t}\right) \exp \left(-\mu_{\mathrm{a}} v t\right)
$$

Consequently, in the presence of scattering changes, the logarithmic ratio becomes

$$
S\left(\lambda_{0}, \lambda, t\right)=\ln \left[\frac{\varphi(\lambda, t)}{\varphi\left(\lambda_{0}, t\right)}\right]=\ln \left[\frac{A(\lambda)}{A\left(\lambda_{0}\right)} \frac{\mu_{\mathrm{s}}^{\prime}}{\mu_{\mathrm{s} 0}^{\prime}}\right]-\frac{3 \rho^{2}}{4 v t} \Delta \mu_{\mathrm{s}}^{\prime}\left(\lambda_{0}, \lambda\right) v t
$$

where $\Delta \mu_{\mathrm{s}}^{\prime}\left(\lambda_{0}, \lambda\right)=\mu_{\mathrm{s}}^{\prime}(\lambda)-\mu_{\mathrm{s}}^{\prime}\left(\lambda_{0}\right)$ is the change in the reduced scattering coefficient between $\lambda_{0}$ and $\lambda$.

The corresponding absorption change $\Delta \mu_{\mathrm{a}}$ is obtained from the time derivative:

$$
\frac{\mathrm{d} S\left(\lambda_{0}, \lambda, t\right)}{\mathrm{d} t}=\frac{3 \rho^{2}}{4 v t^{2}} \Delta \mu_{\mathrm{s}}^{\prime}\left(\lambda_{0}, \lambda\right)-\Delta \mu_{\mathrm{a}}\left(\lambda_{0}, \lambda\right) v
$$

The absorption change $\Delta \mu_{\mathrm{a}}^{*}$, estimated neglecting the scattering changes with wavelength $\left(\Delta \mu_{\mathrm{s}}^{\prime}=0\right)$, derives from the logarithmic ratio as follows:

$$
\frac{\mathrm{d} S\left(\lambda_{0}, \lambda, t\right)}{\mathrm{d} t}=-\Delta \mu_{\mathrm{a}}^{*}\left(\lambda_{0}, \lambda\right) v
$$

Thus, from Eqs. 5 and 6 the absolute error $\varepsilon_{\mathrm{a}}\left(\Delta \mu_{\mathrm{s}}^{\prime}\right)$ in the estimate of the absorption change due to scattering variations $\Delta \mu_{\mathrm{s}}^{\prime}$ can be calculated as

$$
\varepsilon_{\mathrm{a}}\left(\Delta \mu_{\mathrm{s}}^{\prime}\right)=\Delta \mu_{\mathrm{a}}^{*}-\Delta \mu_{\mathrm{a}}=-\frac{3 \rho^{2}}{(2 v t)^{2}} \Delta \mu_{\mathrm{s}}^{\prime}=-K \Delta \mu_{\mathrm{s}}^{\prime}
$$

where

$$
K=\frac{3 \rho^{2}}{(2 v t)^{2}}
$$

is the leverage factor to obtain the error in the absorption change from the scattering change.

The following observations can be made:

(1) The error increases linearly with the scattering changes.

(2) The estimate of strong absorption changes is less affected.

(3) The error is independent of the absolute absorption value $\mu_{\mathrm{a} 0}$. Thus, as an example, when measurements are performed on biological tissues, better results will be 


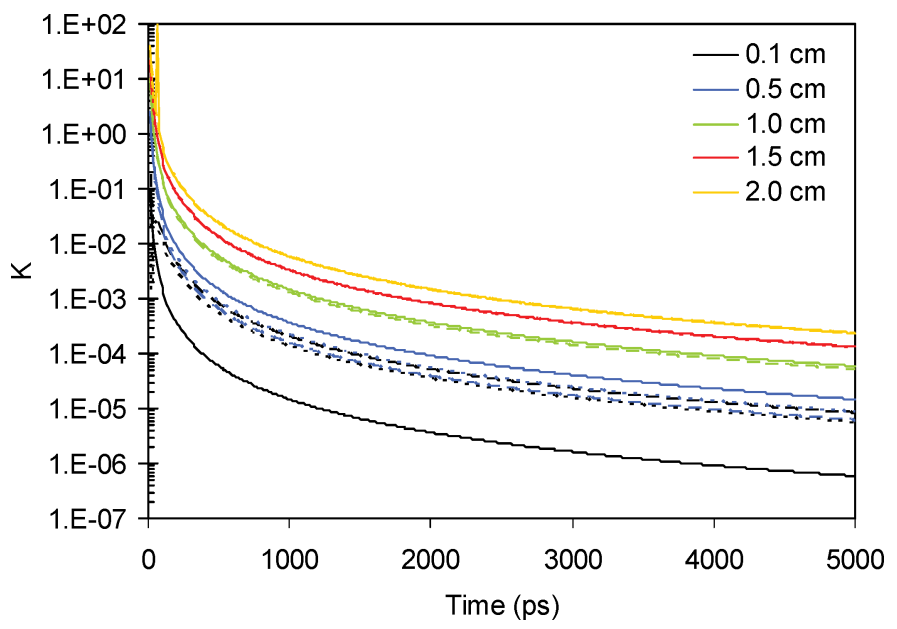

FIg. 1. Factor $K$ versus time $t$ as obtained from Eq. 7 for an infinite homogeneous medium (solid lines) and from the diffusion equation for a semiinfinite homogeneous medium for $\Delta \mu_{\mathrm{s}}^{\prime}=0.5 \mathrm{~cm}^{-1}$ (dashed lines) and $5.0 \mathrm{~cm}^{-1}$ (dotted lines). In all cases, the results are shown for $\rho=0.1,0.5,1.0,1.5$, and 2 cm (different colors; see legend).

obtained in the near-infrared range above $850 \mathrm{~nm}$, where the scattering typically shows a slighter spectral dependence, while the absorption is significant and varies rapidly with wavelength due to the lipid and water content.

(4) Measurements carried out at small inter-fiber distances will be less influenced. Recently, the possibility of performing time-resolved reflection measurements at very small source-detector separation $(<5 \mathrm{~mm})$ was investigated and proved to provide higher spatial resolution and contrast in the localization and characterization of optical inhomogeneities. ${ }^{16}$ This opens an interesting field of application for the differential absorption approach, which is essentially insensitive to scattering changes with wavelength at very small inter-fiber distances.

(5) Finally, short times lead to less accurate results. Thus, the best working condition will be a trade-off between high signal-to-noise ratio available at short times and small error achieved at long times.

To obtain a rough quantitative estimate of the error due to scattering changes, we can consider typical values of the involved parameters. If $t=1000 \mathrm{ps}, \rho=2 \mathrm{~cm}$, and $v=0.03 \mathrm{~cm} /$ ps, then $K \approx 3 \times 10^{-3}$. If $\Delta \mu_{\mathrm{s}}^{\prime}=1 \mathrm{~cm}^{-1}$ in a selected spectral range, then the estimated absorption change $\Delta \mu_{\mathrm{a}}^{*}$ in that range will differ from the real absorption change $\Delta \mu_{\mathrm{a}}$ by approximately $3 \times 10^{-3} \mathrm{~cm}^{-1}$. Considering again biological tissues, and specifically breast tissue, $\Delta \mu_{\mathrm{s}}^{\prime} \cong 1 \mathrm{~cm}^{-1}$ is typically observed on average over the range $900-1000 \mathrm{~nm}$, where the absorption varies from $0.08-0.10 \mathrm{~cm}^{-1}$ to $0.15-0.35 \mathrm{~cm}^{-1}$, depending on breast type. ${ }^{17}$ This would lead to an error of $\sim 1-$ $4 \%$ in the assessment of the peak absorption.

Equation 7 provides a simple means to estimate the absolute error due to scattering changes in cases of practical interest. However, it is an approximated expression. In particular, it relies on the hypothesis of infinite homogeneous medium. To test the validity of Eq. 7 the multiplying factor $K$ in the expression of the absolute error was calculated as a function of time from the diffusion equation for a semi-infinite medium (with extrapolated boundary conditions) for different values of the inter-fiber distance $(\rho=0.1,0.5,1.0,1.5$, and $2.0 \mathrm{~cm})$ and of the scattering change $\left(\Delta \mu_{\mathrm{s}}^{\prime}=0.5\right.$ and $\left.5.0 \mathrm{~cm}^{-1}\right)$. The diffusion equation gives similar results for both values of the scattering change, especially at higher $\rho$ values $(\rho>0.5 \mathrm{~cm})$. As shown in Fig. 1, for $\rho \geq 0.5 \mathrm{~cm}$ the simple approximation provided by Eq. 7 overestimates the error. However, the difference between the two approaches reduces upon increasing $\rho$ and becomes negligible for $\rho=1.5-2.0 \mathrm{~cm}$. It is interesting to note that for very small inter-fiber distances, Eq. 7 provides a clear underestimate of the real error. Still, the error is very small $\left(K<10^{-3}\right.$ for $\left.t>500 \mathrm{ps}\right)$ and likely negligible in practical situations. This supports again the potential effective application of the method to measurements performed at "null" source-detector separation. ${ }^{16}$

Errors in the Estimate of $\mu_{\mathrm{a}}(\lambda)$ : Effect of the Non-ideal Instrument Response Function. The effect of a non-ideal instrument response function (IRF) was investigated again on simulated time-resolved diffuse reflection photon distributions derived using the solution of the diffusion equation under the extrapolated boundary conditions for a semi-infinite medium.

The IRF was first modeled with a Gaussian function of known full width at half-maximum (FWHM). However, in real cases, the IRF often has a relatively small FWHM but is characterized by a long tail, more pronounced than in the case of a Gaussian function. To investigate the effects of this feature, an exponential decay of known time constant was also tested as a model of IRF. For the Gaussian case, the following values were chosen as representative of situations of actual interest: $\mu_{\mathrm{a}}=0.1 \mathrm{~cm}^{-1}, \Delta \mu_{\mathrm{s}}^{\prime}=10 \mathrm{~cm}^{-1}, \rho=1.0 \mathrm{~cm}$, and IRF with $\mathrm{FWHM}=100$ ps. Then each parameter was varied separately to investigate how it affects the relative error $\varepsilon_{\mathrm{r}}(\mathrm{IRF})$ on $\Delta \mu_{\mathrm{a}}$ due to the non-ideal instrument response. The results are reported in Fig. 2. The finite duration of the IRF determines a marked negative error at very short times and a positive error at longer times. Upon increasing the FWHM (Fig. 2a), the effects of the non-ideal behavior increase and extend up to longer times. However, for $t>500 \mathrm{ps}$, the error is always $<3 \%$. A small inter-fiber distance $(\rho<1.0 \mathrm{~cm})$ essentially introduces a positive error, while higher $\rho$ values have the opposite effect. In all cases considered $(\rho=0.1-2.0 \mathrm{~cm})$, the error becomes negligible $(<2 \%)$ for $t>500$ ps (Fig. 2b). A similar trend, but with even smaller values of the corresponding error, is observed upon varying $\mu_{\mathrm{s}}^{\prime}$ (Fig. 2c). Finally, the relative error in the estimate of $\Delta \mu_{\mathrm{a}}$ is essentially independent of the absorption change over the range $\Delta \mu_{\mathrm{a}}=0.02-0.1 \mathrm{~cm}^{-1}$, and it never exceeds $1 \%$ for $t>250$ ps (Fig. 2d).

For the IRF with exponential decay, the following values were chosen as representative of situations of actual interest: $\mu_{\mathrm{a}}$ $=0.1 \mathrm{~cm}^{-1}, \mu_{\mathrm{s}}^{\prime}=10 \mathrm{~cm}^{-1}, \rho=1.0 \mathrm{~cm}$, and IRF with decay constant $\tau=100 \mathrm{ps}$. The situation becomes more critical when the exponentially decaying IRF is considered. If the time constant of the IRF is $\tau=100 \mathrm{ps,} \mathrm{the} \mathrm{relative} \mathrm{error} \mathrm{is} \mathrm{always}$ $\leq 6 \%$, but for $\tau=200$ ps the error on the estimate of $\Delta \mu_{\mathrm{a}}$ becomes exceedingly high at any time (Fig. 3a). The dependence of the error on the inter-fiber distance (Fig. 3b) is qualitatively similar to that already discussed for the Gaussian function, but quantitatively more pronounced. The error becomes $<5 \%$ for $t>500$ ps when $\rho \geq 1.0 \mathrm{~cm}$, while it requires much longer times to become negligible when smaller inter-fiber distances are used. The error is more marked for small scattering values. However, even when $\mu_{\mathrm{s}}^{\prime}=5 \mathrm{~cm}^{-1}$, it becomes $<5 \%$ for $t>800$ ps (Fig. 3c). Finally, upon increasing the estimated absorption change $\Delta \mu_{\mathrm{a}}$, the error tends 

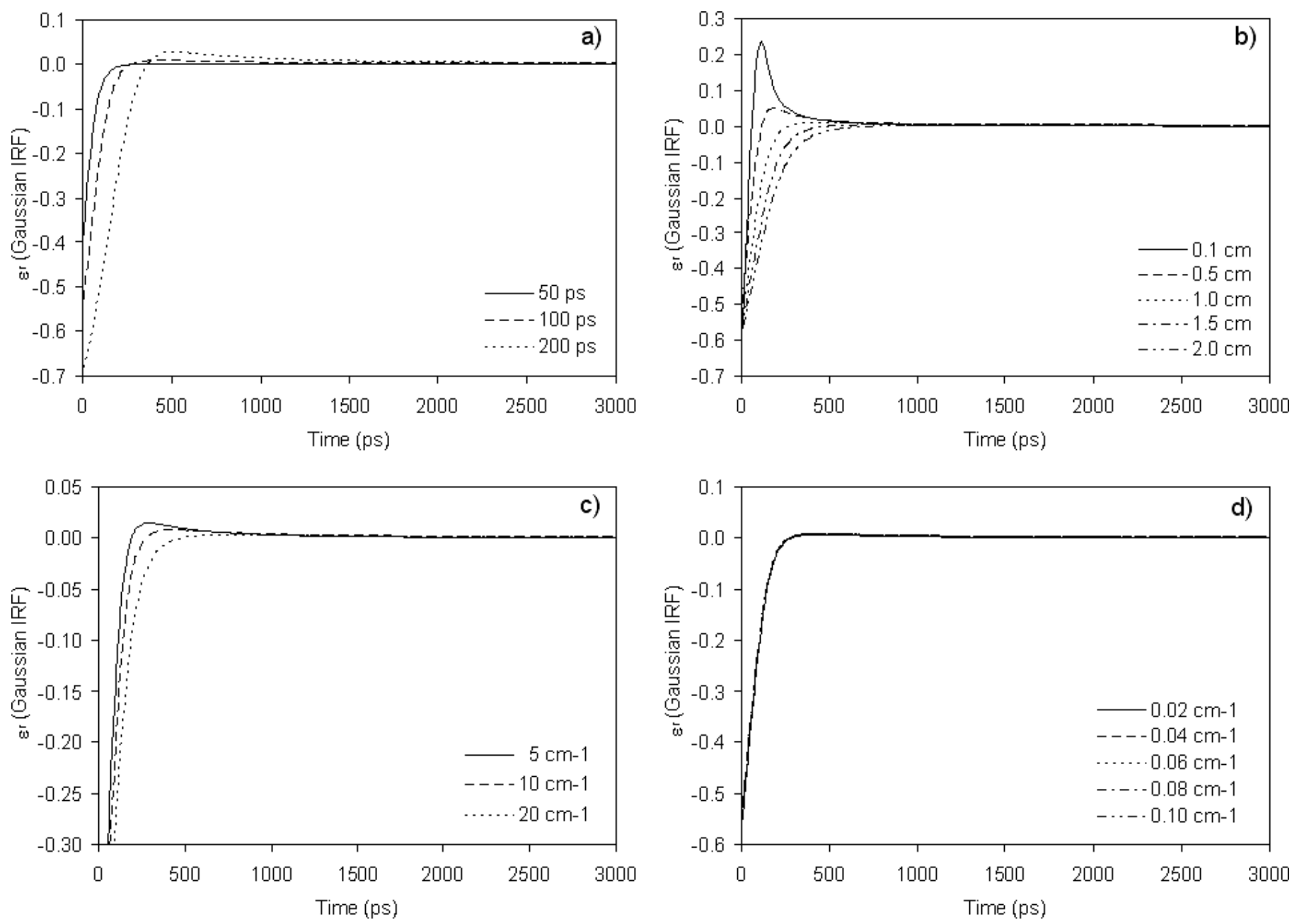

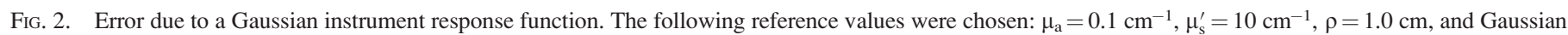

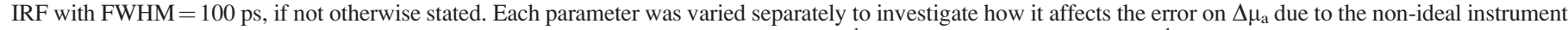
response: (a) FWHM of the IRF $=50-200 \mathrm{ps}$; (b) $\rho=0.1-2.0 \mathrm{~cm} ;(\mathbf{c}) \mu_{\mathrm{s}}^{\prime}=5-20 \mathrm{~cm}^{-1}$; and $(\mathbf{d}) \Delta \mu_{\mathrm{a}}=0.02-0.10 \mathrm{~cm}-1$.

to affect the estimate up to longer times, but it never exceeds $6 \%$ (Fig. 3d). It should be taken into account that, as described previously, the error due to changes in the scattering properties with wavelength more markedly affects the estimate of small absorption changes, opposite to what was just observed for a non-ideal IRF. This reduces the risk that the combined effects of different sources of error yield an overall exceedingly high error in the estimate of the absorption properties. A similar observation holds for the use of small inter-fiber distances that suffer more from a non-ideal IRF, while favored in cases of spectral changes of the scattering coefficient.

In summary, a non-ideal IRF affects the time distribution of re-emitted photons mostly at short times, over a time interval of approximately 500-1000 ps. For longer times, its effects tend to become negligible. It is worth noting that the presence of a long tail seems to be more detrimental for the estimate of $\Delta \mu_{\mathrm{a}}$ than the finite value of the FWHM.

\section{EXPERIMENTAL TEST}

System Setup. The system is fully automated concerning both data acquisition and analysis ${ }^{18}$ and operates continuously between 610 and $1100 \mathrm{~nm}$.

Both optics and alignment of an actively mode-locked
Ti:Sapphire laser were optimized for operation beyond 1000 $\mathrm{nm}$, and it was used to provide picosecond pulses from 900 to $1100 \mathrm{~nm}$. To cover shorter wavelengths (600-900 nm), a supercontinuum fiber source was exploited. Wavelength selection was achieved with the computer-controlled rotation of a Pellin-Broca prism so that the selected wavelength in the dispersed light could be coupled to a $50-\mu \mathrm{m}$ optical fiber, placed distal to the prism. ${ }^{19}$

Optical fibers delivered the illumination light to the sample and collected the diffusely reflected light. Time-correlated single-photon counting was used for the detection.

The overall time resolution of the system is 70-160 ps, depending on wavelength. For the present study, time-resolved data were collected every $5 \mathrm{~nm}$, with an acquisition time of $4 \mathrm{~s}$ per wavelength.

Experimental Measurements. To test the effect of the inter-fiber distance in critical conditions for method applicability, spectral time-resolved reflection measurements were performed on a resin tissue phantom every $5 \mathrm{~nm}$ from 620 to $890 \mathrm{~nm}$. The inter-fiber distance $\rho$ was varied between 1 and 3 $\mathrm{cm}(\rho=1.0,1.5,2.0,2.5$, and $3.0 \mathrm{~cm}) . \lambda_{0}=780 \mathrm{~nm}$ was chosen as a reference for the estimate of absorption changes. The results are displayed in Fig. 4. The resin phantom is characterized by a limited variation of the absorption properties 

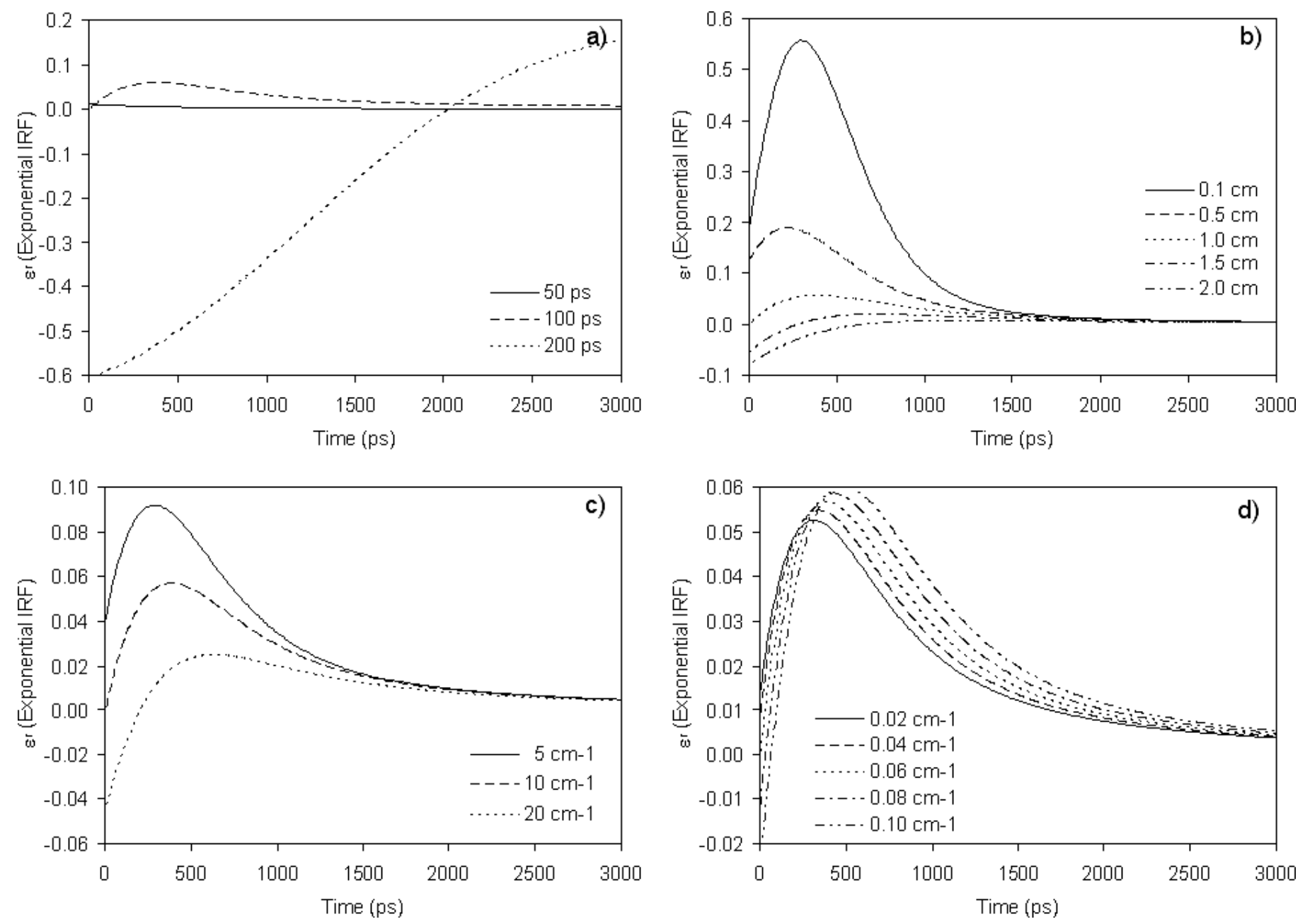

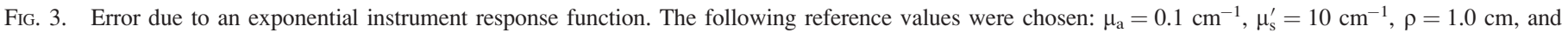

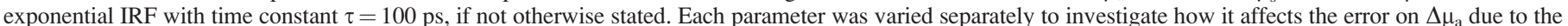
non-ideal instrument response: (a) decay constant $\tau$ of the IRF $=50-200 \mathrm{ps} ;(\mathbf{b}) \rho=0.1-2.0 \mathrm{~cm}^{\prime}(\mathbf{c}) \mu_{\mathrm{s}}^{\prime}=5-20 \mathrm{~cm}^{-1} ;$ and $(\mathbf{d}) \Delta \mu_{\mathrm{a}}=0.02-0.10 \mathrm{~cm}^{-1}$.

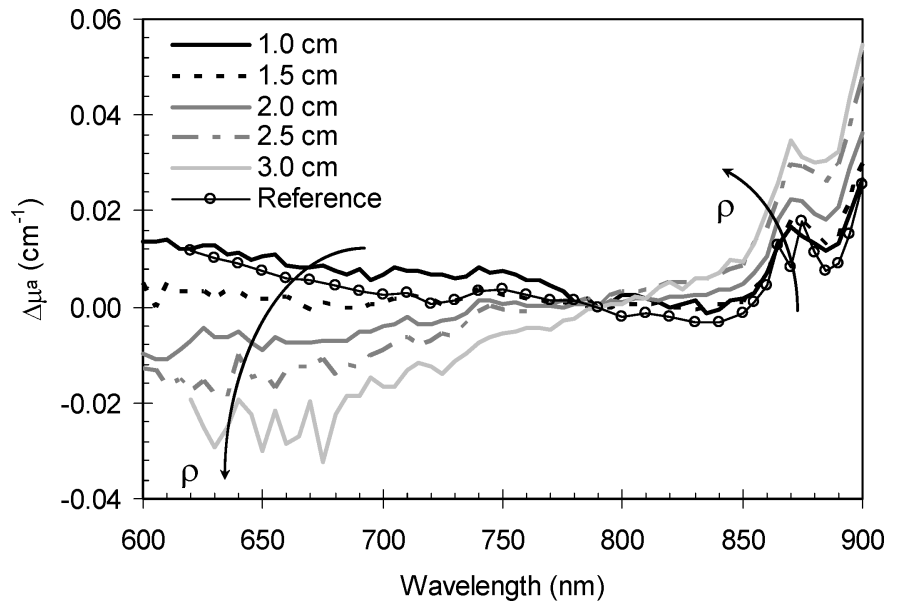

FIG. 4. $\Delta \mu_{\mathrm{a}}$ versus wavelength for a resin tissue phantom, estimated for different values of the inter-fiber distance $\rho=1.0,1.5,2.0,2.5$, and $3.0 \mathrm{~cm}$ (lines). As a reference, the results obtained with a conventional fit to the diffusion equation are also reported (symbols). over the measurement range $\left(\Delta \mu_{\mathrm{a}} \cong 0.02 \mathrm{~cm}^{-1}\right)$, while the reduced scattering decreases significantly upon increasing wavelength $\left(\Delta \mu_{\mathrm{s}}^{\prime} \cong 4.5 \mathrm{~cm}^{-1}\right)$. Both these features make the measurement critical and contribute to increase the error in the estimate of the absorption, as highlighted by Eq. 7. This notwithstanding, if a small value of the inter-fiber distance is exploited $(\rho=1 \mathrm{~cm})$, the absorption line shape can be estimated. As expected, the error increases progressively upon increasing $\rho$ value, with a maximum error on $\Delta \mu_{\mathrm{a}}$ always $<0.03 \mathrm{~cm}^{-1}$.

The insensitivity of the method to the sample size and specifically to the effect of close sample boundaries was tested by performing measurements on wet wood samples of interest for practical applications. ${ }^{20}$ The samples were parallelepipeds of fir wood $(3.2 \mathrm{~cm} \mathrm{~W} \times 1.8 \mathrm{~cm} \mathrm{D} \times 2.5 \mathrm{~cm} \mathrm{H})$. The inter-fiber distance was set to $\rho=1.0 \mathrm{~cm}$. Time-resolved data were collected every $5 \mathrm{~nm}$ from 910 to $1040 \mathrm{~nm}$, placing the fibers perpendicular to the top surface and in contact with it: (1) at the center of the sample side, and (2) near the sample boundary, so that the collection fiber was actually located at the side edge, while the injection fiber was $1.0 \mathrm{~cm}$ away from it. Due to its microscopic structure with aligned fibers, wood is a markedly anisotropic material. Considerably different reduced scattering properties are estimated when measurements are performed 
parallel (par) to the fiber direction as compared to perpendicular to it (per). For the wood samples considered in the present study, $\mu_{\mathrm{s}}^{\prime} \cong 15 \mathrm{~cm}^{-1}$ in the former geometry and $\mu_{\mathrm{s}}^{\prime} \cong 100$ $\mathrm{cm}^{-1}$ in the latter one, essentially independent of wavelength, as the scattering spectra are fairly flat. To test possible effects of anisotropy on the estimate of $\Delta \mu_{\mathrm{a}}$, measurements were performed at the center of the sample in par geometry.

A reference wavelength $\lambda_{0}=940 \mathrm{~nm}$ was chosen for the estimate of $\Delta \mu_{\mathrm{a}}$. The corresponding absorption line shapes are displayed in Fig. 5. For comparison, a measurement performed at the center of the sample was also fitted with a conventional procedure that estimates $\mu_{\mathrm{a}}$ and $\mu_{\mathrm{s}}^{\prime}$ relying on the diffusion equation with extrapolated boundary conditions, ${ }^{1,21}$ and the result is displayed in Fig. 5. Just one configuration is considered as a reference (i.e., per geometry), because the diffusion theory for an isotropic medium allows the correct assessment of the absorption properties of anisotropic media when time-resolved measurements are interpreted, leading to the same results for both par and per configurations. ${ }^{22}$ To evaluate the performance of the proposed method, let us first consider data collected in per geometry placing the fibers in different positions. When the measurement is performed at the center of the sample side, the estimated absorption properties are very close to those obtained with the conventional fitting procedure. Satisfactory results are still obtained when $\Delta \mu_{\mathrm{a}}$ is estimated from data collected at the edge of the sample side: in the peak range $(990-1010 \mathrm{~nm})$ it is on average only $6.7 \%$ lower. This confirms that the estimated $\Delta \mu_{\mathrm{a}}$ is not significantly affected by boundary effects.

The average difference between the par measurement and the corresponding per measurement in the peak range (990$1010 \mathrm{~nm}$ ) is $15.3 \%$. It should be taken into account that, at least in part, the difference between the absorption line shapes estimated in distinct geometries (center versus edge of the sample, parallel versus perpendicular to the fiber direction) may well be due to local heterogeneity in the optical properties of wood. However, as compared to the per measurement carried out at the center of the sample, the time distribution of the reflected photons is much narrower for both the measurement performed at the edge and the one in par geometry. This limits the time $t$ for data interpretation and, as discussed above, is consequently expected to lead to a higher error in the estimate of the absorption change, in agreement with what is shown in Fig. 5.

\section{CONCLUSION}

We have proposed a method for the estimate of the absorption line shape of highly diffusive media. The same general principle was previously proposed to assess the absorption change between two wavelengths and then derive the absolute concentrations of oxy- and deoxyhemoglobin, considered as the only tissue absorbers. ${ }^{14} \mathrm{We}$ extended the application of that principle to a full wavelength range with the aim of estimating the spectral dependence of the absorption over that range. The method is based on the Beer-Lambert law. It is thus essentially insensitive to the sample geometry (size and shape), and it does not need to fulfill the strict hypotheses of the diffusion approximation. Moreover, it proved to be only relatively affected by the sample anisotropy.

The proposed differential absorption approach is expected to be intrinsically more stable than conventional fitting methods

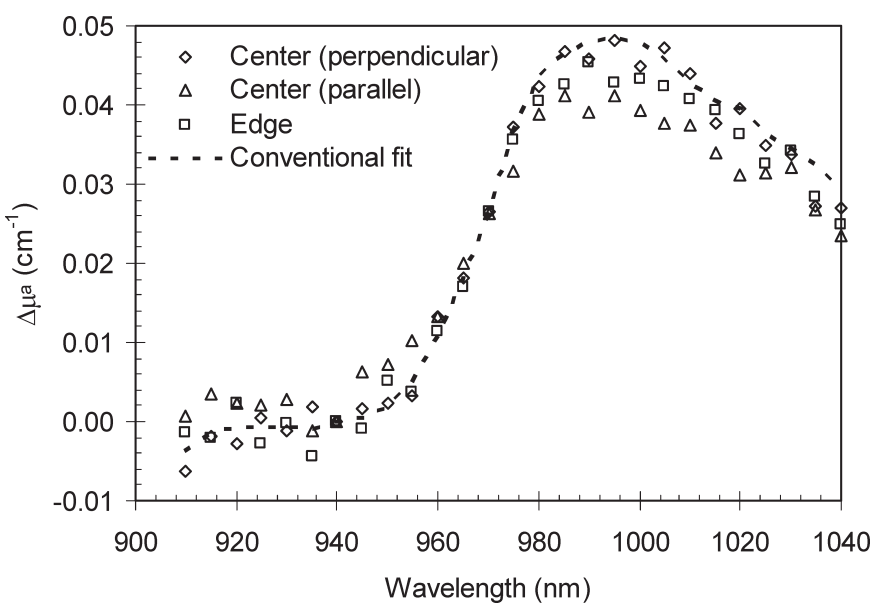

FIG. 5. $\Delta \mu_{\mathrm{a}}$ versus wavelength for silver fir. As detailed in the text, measurements were performed in various configurations: center-perpendicular (diamonds), center-parallel (triangles), and edge-perpendicular (squares). As a reference, the results obtained with a conventional fit to the diffusion equation are also reported (center-perpendicular configuration, dashed line).

based on the solution of the diffusion equation, as it relies on a linear procedure as compared to nonlinear inversion ones.

Only a limiting hypothesis is made, regarding the wavelength independence of the scattering properties. The error made when this hypothesis is not (or not fully) fulfilled was shown to be lower when the absorption changes rapidly with wavelength, measurements are performed with smaller separation between light injection and collection points, and the data are interpreted at long times.

The non-ideal IRF introduces an error with value and sign depending on the measurement conditions, but it is generally negligible for $t>500-1000 \mathrm{ps}$, in agreement with the choice of long times to minimize the error due to spectral changes in the scattering properties. If both the FWHM of the IRF and the slope of its trailing edge are considered, the latter seems to be a stronger cause of non-ideality.

To summarize, in practical measurement conditions, two contributions can be identified in the absolute error that affect the estimate of $\Delta \mu_{\mathrm{a}}$ :

$$
\varepsilon_{\mathrm{a}}=-K \Delta \mu_{\mathrm{s}}^{\prime}+\varepsilon_{\mathrm{r}}(\mathrm{IRF}) \Delta \mu_{\mathrm{a}}
$$

It is noteworthy that the method was introduced and investigated for application in reflection geometry, but all considerations can easily be extended to measurements performed in transmission geometry, and in general to any other situation, as long as a homogeneous medium is considered.

A second method, related to the one proposed here, is also under investigation. It exploits information on the amplitude $A(\lambda)$, obtained through a preliminary calibration on a medium of known optical properties, to reduce to a single time point, instead of the entire time distribution, the information needed to estimate $\Delta \mu_{\mathrm{a}}$. This would lead to experimentally simpler measurements.

Finally, for wider applicability, the proposed method could in principle also be extended to heterogeneous media. The real applicability and performance of the differential absorption approach in the case of heterogeneous media will be investigated in the near future. 


\section{ACKNOWLEDGMENT}

The research leading to these results has received funding from the EC's Seventh Framework Programme (FP7/2007-2013) under grant agreements No. 228334 and No. 201076.

1. M. S. Patterson, B. Chance, and B. C. Wilson, Appl. Opt. 28, 2331 (1989).

2. J. C. J. Paasschens, Phys. Rev. E 56, 1135 (1997).

3. F. Martelli, A. Sassaroli, A. Pifferi, A. Torricelli, L. Spinelli, and G. Zaccanti, Opt. Exp. 15, 18168 (2007).

4. F. Martelli, S. Del Bianco, A. Ismaelli, and G. Zaccanti, Light Propagation through Biological Tissue and Other Diffusive Media: Theory, Solutions, and Software (SPIE Press, Bellingham, 2009).

5. S. R. Arridge, M. Cope, and D. T. Delpy, Phys. Med. Biol. 37, 1531 (1992).

6. A. Kienle, J. Opt. Soc. Am. A 22, 1883 (2005)

7. E. Margallo-Balbás and P. J. French, Opt. Exp. 15, 14086 (2007).

8. R. Lu, D. E. Guyer, and R. M. Beaudry, J. Text. Studies 31, 615 (2000)

9. H. Schulz, M. Baranska, and R. Baranski, Biopolymers 77, 212 (2005).

10. B. M. Nicolaï, B. E. Verlinden, M. Desmet, S. Saevels, W. Saeys, K. Theron, R. Cubeddu, A. Pifferi, and A. Torricelli, Postharvest Bio. Technol. 47, 68 (2008).
11. A. Torricelli, L. Spinelli, D. Contini, M. Vanoli, A. Rizzolo, and P. Eccher Zerbini, Sens. Instrum. Food Qual. 2, 82 (2008).

12. A. A. Gowen, C. P. O'Donnell, P. J. Cullen, and S. E. J. Bell, Eur. J. Pharm. Biopharm. 69, 10 (2008).

13. G. Reich, Adv. Drug Deliv. Rev. 57, 1109 (2005).

14. M. Oda, Y. Yamashita, G. Nishimura, and M. Tamura, Phys. Med. Biol. 41, 551 (1996).

15. C. D'Andrea, L. Spinelli, A. Bassi, A. Giusto, D. Contini, J. Swartling, A. Torricelli, and R. Cubeddu, Opt. Exp. 5, 1888 (2006).

16. L. Spinelli, F. Martelli, S. Del Bianco, A. Pifferi, A. Torricelli, R. Cubeddu, and G. Zaccanti, Phys. Rev. E 74, 021919 (2006).

17. A. Pifferi, J. Swartling, E. Chikoidze, A. Torricelli, P. Taroni, A. Bassi, S. Andersson-Engels, and R. Cubeddu, J. Biomed. Opt. 9, 1143 (2004)

18. A. Pifferi, A. Torricelli, P. Taroni, D. Comelli, A. Bassi, and R. Cubeddu, Rev. Sci. Instrum. 78, 053103 (2007).

19. A. Bassi, A. Farina, C. D'Andrea, A. Pifferi, G. Valentini, and R. Cubeddu, Opt. Exp. 15, 14482 (2007).

20. C. D'Andrea, A. Farina, D. Comelli, A. Pifferi, P. Taroni, G. Valentini, and R. Cubeddu, Appl. Spectrosc. 62, 569 (2008).

21. R. C. Haskell, L. O. Svasaand, T. T. Tsay, T. C. Feng, M. S. McAdams, and B. J. Tromberg, J. Opt. Soc. Am. A 11, 2727 (1994).

22. A. Kienle, C. D’Andrea, F. Foschum, P. Taroni, and A. Pifferi, Opt. Exp. 16, 9895 (2008) 